\title{
Finite automata for Schreier graphs of virtually free groups
}

\author{
Pedro V. Silva, Xaro Soler-Escrivà and Enric Ventura \\ Communicated by James Howie
}

\begin{abstract}
The Stallings construction for f.g. subgroups of free groups is generalized by introducing the concept of Stallings section, which allows efficient computation of the core of a Schreier graph based on edge folding. It is proved that the groups that admit Stallings sections are precisely the f.g. virtually free groups, this is proved through a constructive approach based on Bass-Serre theory. Complexity issues and applications are also discussed.
\end{abstract}

\section{Introduction}

Finite automata have, over the years, become the standard representation of finitely generated subgroups $H$ of a free group $F_{A}$. The Stallings construction constitutes a simple and efficient algorithm for building an automaton $\$(H)$ which can be used for solving the membership problem of $H$ in $F_{A}$ and many other applications. This automaton $\delta(H)$ is nothing more than the core automaton of the Schreier graph (automaton) of $H$ in $F_{A}$, whose structure can be described as $\delta(H)$ with finitely many infinite trees adjoined. Many features of $\delta(H)$ were (re)discovered over the years and were known to Reidemeister, Schreier, and particularly Serre [17]. One of the greatest contributions of Stallings [19] is certainly the algorithm to construct $\delta(H)$ : taking a finite set of generators $h_{1}, \ldots, h_{m}$ of $H$ in reduced form, we start with the so-called flower automaton, where petals

The first named author was partially supported by CMUP (UID/MAT/00144/2013), which is funded by FCT (Portugal) with national (MEC) and European structural funds through the programs FEDER, under the partnership agreement PT2020. The second named author acknowledges support from Proyecto MTM2010-19938-C03-01 of Ministerio de Ciencia e Innovación of Spain. The third named author acknowledges partial support from the Spanish Government through project number MTM2011-25955. 
labelled by the words $h_{i}$ (and their inverse edges) are glued to a basepoint $q_{0}$ :

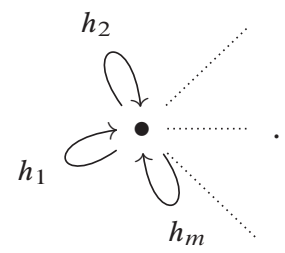

Then we proceed by successively folding pairs of edges of the form $q \stackrel{a}{\leftarrow} p \stackrel{a}{\rightarrow} r$ until no more folding is possible (so we get an inverse automaton). And we will have just built $\delta(H)$. For details and applications of the Stallings construction, see $[1,6,13]$.

Since $8(H)$ turns out to be the core of the Schreier graph of $H \leq F_{A}$, this construction is independent of the finite set of generators of $H$ chosen at the beginning, and of the particular sequence of foldings followed. And the membership problem follows from the fact that $\delta(H)$ recognizes all the reduced words representing elements of $H, \ldots$ and the reduced words constitute a section for any free group.

Such an approach naturally invites generalizations for further classes of groups. For instance, an elegant geometric construction of Stallings type automata was achieved for amalgams of finite groups by Markus-Epstein [12]. On the other hand, the most general results were obtained by Kapovich, Weidmann and Miasnikov [7] for finite graphs of groups where each vertex group is either polycyclic-by-finite or word-hyperbolic and locally quasiconvex, and where all edge groups are virtually polycyclic. However, the complex algorithms were designed essentially to solve the generalized word problem, and it seems very hard to extend other features of the free group case, either geometric or algorithmic. Our goal in the present paper is precisely to develop a Stallings type approach with some generality which is robust enough to exhibit several prized algorithmic and geometric features, namely in connection with Schreier graphs. Moreover, we are able to identify those groups $G$ for which this can be achieved: (finitely generated) virtually free groups.

What ingredients do we need to get a Stallings type algorithm? First of all, we need a section $S$ with good properties that can emulate the role played by the reduced words in the free group. In particular, we need a rational language (i.e. recognizable by a finite automaton). We may of course need to be more restrictive than taking all reduced words, if we want our finite automaton to recognize all the representatives of $H \leq_{f . g}$. $G$ in $S$. To get inverse automata, it is also convenient to have $S=S^{-1}$

Secondly, the set $S_{g}$ of words of $S$ representing a certain $g \in G$ must be at least rational, so we can get a finite automaton to represent each of the generalized 
petals. Third, the folding process to be performed in the (generalized) flower automaton (complemented possibly by other identification operations) must ensure in the end that all representatives of elements of $H$ in $S$ are recognized by the automaton. And folding is the automata-theoretic translation of the reduction process $w \rightarrow \bar{w}$ taking place in the free group. So we need the condition $S_{g_{1} g_{2}} \subseteq \overline{S_{g_{1}} S_{g_{2}}}$, to make sure that the petals (corresponding to the generators of $H$ ) carry enough information to produce, after the subsequent folding, all the representatives of elements of $H$. This is how we were led to our definition of Stallings section.

It is somewhat surprising how much we can get from this concept, which turns out to be more robust than one would expect. Among other features, we can mention independence from the generating set (so we can have Stallings automata for free groups when we consider a noncanonical generating set!), or closure of rational sets with respect to computation of normal forms. We present some applications of the whole theory, believing that many others should follow in due time, as happened in the free group case.

The paper is structured as follows. In Section 2 we present the necessary basic concepts. The theory of Stallings sections is presented in Section 3. In Section 4, we discuss the complexity of the generalized Stallings construction in its most favourable version. In Section 5 we use Muller-Schupp's Theorem and Bass-Serre theory to prove that those groups admitting a Stallings section are precisely the finitely generated virtually free groups. In Section 6 we show that we can assume stronger properties for Stallings sections with an eye to applications, namely the characterization of finite index subgroups.

\section{Preliminaries}

Given a finite alphabet $A$, we denote by $A^{*}$ the free monoid on $A$, with 1 denoting the empty word. A subset of a free monoid is called a language.

We say that $\mathcal{A}=\left(Q, q_{0}, T, E\right)$ is a (finite) $A$-automaton if:

- $Q$ is a (finite) set,

- $q_{0} \in Q$ and $T \subseteq Q$,

- $E \subseteq Q \times A \times Q$.

A nontrivial path in $\mathcal{A}$ is a sequence

$$
p_{0} \stackrel{a_{1}}{\longrightarrow} p_{1} \stackrel{a_{2}}{\longrightarrow} \cdots \stackrel{a_{n}}{\longrightarrow} p_{n}
$$

with $\left(p_{i-1}, a_{i}, p_{i}\right) \in E$ for $i=1, \ldots, n$. Its label is the word

$$
a_{1} \cdots a_{n} \in A^{+}=A^{*} \backslash\{1\} .
$$

It is said to be a successful path if $p_{0}=q_{0}$ and $p_{n} \in T$. We consider also the 
trivial path $p \stackrel{1}{\rightarrow} p$ for $p \in Q$. It is successful if $p=q_{0} \in T$. The language $L(\mathcal{A})$ recognized by $\mathcal{A}$ is the set of all labels of successful paths in $\mathcal{A}$. A path of minimal length between two vertices is called a geodesic, and so is its label by extension.

The automaton $\mathcal{A}=\left(Q, q_{0}, T, E\right)$ is said to be deterministic if, for all $p \in Q$ and $a \in A$, there is at most one edge of the form $(p, a, q)$. We say that $\mathcal{A}$ is trim if every $q \in Q$ lies in some successful path.

Given deterministic $A$-automata $\mathcal{A}=\left(Q, q_{0}, T, E\right)$ and $\mathcal{A}^{\prime}=\left(Q^{\prime}, q_{0}^{\prime}, T^{\prime}, E^{\prime}\right)$, a morphism $\varphi: \mathcal{A} \rightarrow \mathcal{A}^{\prime}$ is a mapping $\varphi: Q \rightarrow Q^{\prime}$ such that

- $q_{0} \varphi=q_{0}^{\prime}$ and $T \varphi \subseteq T^{\prime}$,

- $(p \varphi, a, q \varphi) \in E^{\prime}$ for every $(p, a, q) \in E$.

It follows that $L(\mathcal{A}) \subseteq L\left(\mathcal{A}^{\prime}\right)$ if there is a morphism $\varphi: \mathcal{A} \rightarrow \mathcal{A}^{\prime}$. The morphism $\varphi: \mathcal{A} \rightarrow \mathcal{A}^{\prime}$ is:

- injective if it is injective as a mapping $\varphi: Q \rightarrow Q^{\prime}$,

- an isomorphism if it is injective, $T^{\prime}=T \varphi$ and every edge of $E^{\prime}$ is of the form $(p \varphi, a, q \varphi)$ for some $(p, a, q) \in E$.

The star operator on $A$-languages is defined by

$$
L^{*}=\bigcup_{n \geq 0} L^{n},
$$

where $L^{0}=\{1\}$. A language $L \subseteq A^{*}$ is said to be rational (or to admit a rational expression) if $L$ can be obtained from finite languages using the operators union, product and star a finite number of times. Alternatively, $L$ is rational if and only if it is recognized by a finite (deterministic) $A$-automaton $\mathcal{A}=\left(Q, q_{0}, T, E\right)$, see $[3$, Section III]. The definition generalizes to subsets of an arbitrary monoid $M$ in the obvious way.

We denote the set of all rational languages $L \subseteq A^{*}$ by Rat $A^{*}$. Note that Rat $A^{*}$, endowed with the product of languages, constitutes a monoid.

In the statement of a result, we shall say that a rational language $L$ is effectively constructible if there exists an algorithm to produce from the data implicit in the statement a finite $A$-automaton $\mathcal{A}$ recognizing $L$.

It is convenient to summarize some closure and decidability properties of rational languages in the following proposition (see, e.g., [3]). The prefix set of a language $L \subseteq A^{*}$ is defined as

$$
\operatorname{Pref}(L)=\left\{u \in A^{*} \mid u A^{*} \cap L \neq \emptyset\right\} .
$$

A rational substitution is a morphism $\varphi: A^{*} \rightarrow$ Rat $B^{*}$ (where Rat $B^{*}$ is endowed 
with the product of languages). Given $L \subseteq A^{*}$, we denote by $L \varphi$ the language

$$
\bigcup_{u \in L} u \varphi \subseteq B^{*} .
$$

Since singletons are rational languages, monoid homomorphisms constitute particular cases of rational substitutions.

More generally, a mapping $\tau: A^{*} \rightarrow 2^{B^{*}}$ is called a transduction. Its graph is defined by

$$
\Delta_{\tau}=\left\{(u, v) \in A^{*} \times B^{*} \mid v \in u \tau\right\} .
$$

The transduction $\tau$ is rational if $\Delta_{\tau}$ is a rational subset of the monoid $A^{*} \times B^{*}$. Rational substitutions constitute a particular case of rational transductions. Rational transductions are most commonly defined through rational transducers, i.e. finite automata with edges labelled by elements of $A \times$ Rat $B^{*}$.

The inverse transduction $\tau^{-1}: B^{*} \rightarrow 2^{A^{*}}$ is defined by

$$
v \tau^{-1}=\left\{u \in A^{*} \mid v \in u \tau\right\} .
$$

It is well known (see [3, Section III.4]) that rational transductions are closed under inversion.

Proposition 2.1. Let $A$ be a finite alphabet and let $K, L \subseteq A^{*}$ be rational. Then:

(i) $K \cup L, K \cap L, A^{*} \backslash L, \operatorname{Pref}(L)$ are rational,

(ii) if $\tau: A^{*} \rightarrow 2^{B^{*}}$ is a rational transduction, then $L \tau$ is rational,

(iii) if $\varphi: A^{*} \rightarrow M$ is a monoid homomorphism and $M$ is finite, then $X \varphi^{-1}$ is rational for every $X \subseteq M$.

Moreover, all the constructions are effective, and the inclusion $K \subseteq L$ is decidable.

The class of context-free languages (see [3, Chapter II] for details) constitutes the next level in the classical Chomsky hierarchy above rational languages. We have also the following closure property (see [3, Corollary III.4.2]):

Proposition 2.2. Let $\tau: A^{*} \rightarrow 2^{B^{*}}$ be a rational transduction and let $L \subseteq A^{*}$ be context-free. Then $L \tau$ is context-free.

Given an $A$-automaton $\mathcal{A}$ and $L \subseteq A^{*}$, we denote by $\mathcal{A} \sqcap L$ the $A$-automaton obtained by removing from $\mathcal{A}$ all the vertices and edges which do not lie in some successful path labelled by a word in $L$.

Proposition 2.3. Let $\mathcal{A}$ be a finite $A$-automaton and let $L \subseteq A^{*}$ be a rational language. Then $\mathcal{A} \sqcap L$ is effectively constructible. 
Proof. Write $\mathcal{A}=\left(Q, q_{0}, T, E\right)$ and let $\mathcal{A}^{\prime}=\left(Q^{\prime}, q_{0}^{\prime}, T^{\prime}, E^{\prime}\right)$ be a finite $A$-automaton recognizing $L$. The direct product

$$
\mathcal{A}^{\prime \prime}=\left(Q \times Q^{\prime},\left(q_{0}, q_{0}^{\prime}\right), T \times T^{\prime}, E^{\prime \prime}\right)
$$

is defined by

$$
E^{\prime \prime}=\left\{\left(\left(p, p^{\prime}\right), a,\left(q, q^{\prime}\right)\right) \mid(p, a, q) \in E,\left(p^{\prime}, a, q^{\prime}\right) \in E^{\prime}\right\} .
$$

Let $\mathscr{B}$ denote the trim part of $\mathcal{A}^{\prime \prime}$ (by removing all vertices/edges which are not part of successful paths in $\mathcal{A}^{\prime \prime}$; this can be done effectively). Then, $\mathcal{A} \sqcap L$ can be obtained by projecting onto the first component the various constituents of $\mathscr{B}$.

Given an alphabet $A$, we denote by $A^{-1}$ the set of formal inverses of $A$, and write $\widetilde{A}=A \cup A^{-1}$. We say that $\widetilde{A}$ is an involutive alphabet. We extend

$$
{ }^{-1}: A \rightarrow A^{-1}, \quad a \mapsto a^{-1},
$$

to an involution on $\widetilde{A}^{*}$ through

$$
\left(a^{-1}\right)^{-1}=a, \quad(u v)^{-1}=v^{-1} u^{-1} \quad\left(a \in A, u, v \in \widetilde{A}^{*}\right) .
$$

An automaton $\mathcal{A}$ over an involutive alphabet $\widetilde{A}$ is said to be involutive if, whenever $(p, a, q)$ is an edge of $\mathcal{A}$, so is $\left(q, a^{-1}, p\right)$. Therefore it suffices to depict just the positively labelled edges (having label in $A$ ) in their graphical representation.

An involutive automaton is inverse if it is deterministic, trim and has a single final state (note that for involutive automata, being trim is equivalent to being connected). If the latter happens to be the initial state, it is called the basepoint.

The next result is folklore. For a proof, see [1, Proposition 2.2].

Proposition 2.4. Given inverse automata $\mathcal{A}$ and $\mathcal{A}^{\prime}$, then $L(\mathcal{A}) \subseteq L\left(\mathcal{A}^{\prime}\right)$ if and only if there exists a morphism $\varphi: \mathcal{A} \rightarrow \mathcal{A}^{\prime}$. Moreover, such a morphism is unique.

Given an alphabet $A$, let $\sim$ denote the congruence on $\widetilde{A}^{*}$ generated by the relation

$$
\left\{\left(a a^{-1}, 1\right) \mid a \in \widetilde{A}\right\} .
$$

The quotient $F_{A}=\widetilde{A}^{*} / \sim$ is the free group on $A$. We denote by $\theta: \widetilde{A}^{*} \rightarrow F_{A}$ the canonical morphism $u \mapsto[u]_{\sim}$.

Alternatively, we can view (2.1) as a confluent length-reducing rewriting system on $\widetilde{A}^{*}$, where each word $w \in \widetilde{A^{*}}$ can be transformed into a unique reduced word $\bar{w}$ with no factor of the form $a a^{-1}$. As a consequence, the equivalence

$$
u \sim v \Longleftrightarrow \bar{u}=\bar{v} \quad\left(u, v \in \widetilde{A}^{*}\right)
$$

solves the word problem for $F_{A}$. We shall use the notation $R_{A}=\widetilde{\widetilde{A}^{*}}$. 
We close this section with the following equivalent version of Benois' Theorem, relating rational languages with free group reduction:

Theorem 2.5 (Benois [2]). If $L \subseteq \widetilde{A}^{*}$ is rational, then $\bar{L}$ is an effectively constructible rational language.

\section{Stallings sections}

Let $G$ be a (finitely generated) group generated by the finite set $A$. More precisely, we consider an epimorphism $\pi: \widetilde{A}^{*} \rightarrow G$ satisfying

$$
a^{-1} \pi=(a \pi)^{-1}
$$

for every $a \in A$. A homomorphism satisfying condition (3.1) is said to be matched. Note that in this case (3.1) holds for arbitrary words. For short, we shall refer to a matched epimorphism $\pi: \widetilde{A}^{*} \rightarrow G$ (with $A$ finite) as an $m$-epi.

We shall call a language $S \subseteq \widetilde{A}^{*}$ a section (for $\pi$ ) if $S \pi=G$ and $S^{-1}=S$. For every $X \subseteq G$, we write

$$
S_{X}=X \pi^{-1} \cap S
$$

We say that an effectively constructible rational section $S \subseteq R_{A}$ is a Stallings section for $\pi$ if, for all $g, h \in G$ :

(S1) $S_{g}$ is an effectively constructible rational language,

(S2) $S_{g h} \subseteq \overline{S_{g} S_{h}}$.

Note that (S2) immediately yields

$$
S_{g_{1} \cdots g_{n}} \subseteq \overline{S_{g_{1}} \cdots S_{g_{n}}}
$$

for all $g_{1}, \ldots, g_{n} \in G$. Moreover, in (S1) it suffices to consider $S_{a \pi}$ for $a \in A$. Indeed, by (3.2), and since $S^{-1}=S$ and $S_{g} \pi=g$ for every $g \in G$, we may write

$$
S_{\left(a_{1} \cdots a_{n}\right) \pi}=\overline{S_{a_{1} \pi} \cdots S_{a_{n} \pi}} \cap S
$$

and

$$
S_{a_{i}^{-1} \pi}=S_{a_{i} \pi}^{-1}
$$

for all $a_{i} \in \widetilde{A}$. Then, by Proposition 2.1 and Theorem $2.5, S_{g}$ is a rational language for every $g \in G$; furthermore, it is effectively constructible from $S_{a_{1} \pi}, \ldots, S_{a_{n} \pi}$.

Note that if $S$ is a Stallings section, then $S \cup\{1\}$ is also a Stallings section. Indeed, it is easy to see that conditions (S1) and (S2) are still verified: namely, if $g h=1$, then $1 \in \overline{S_{g} S_{g}^{-1}}=\overline{S_{g} S_{h}}$ and so $S_{g h} \cup\{1\} \subseteq \overline{S_{g} S_{h}}$ as required.

The next result shows that the existence of a Stallings section is independent from the finite set $A$ and the m-epi $\pi: \widetilde{A}^{*} \rightarrow G$ considered. 
Proposition 3.1. Let $\pi: \widetilde{A}^{*} \rightarrow G$ and $\pi^{\prime}: \widetilde{A}^{\prime *} \rightarrow G$ be two m-epis. Then, $G$ has a Stallings section for $\pi$ if and only if $G$ has a Stallings section for $\pi^{\prime}$.

Proof. Let $S \subseteq R_{A}$ be a Stallings section for $\pi$. There exists an m-epi

$$
\varphi: \widetilde{A}^{*} \rightarrow \widetilde{A}^{\prime *}
$$

such that $\varphi \pi^{\prime}=\pi$. Write $S^{\prime}=\overline{S \varphi}$. By Proposition 2.1 (ii) and Theorem 2.5, $S^{\prime}$ is an effectively constructible rational subset of $R_{A^{\prime}}$. We claim that

$$
S_{g}^{\prime}=\overline{S_{g} \varphi}
$$

holds for every $g \in G$.

Indeed, let $u \in S_{g}^{\prime}$. Then $u=\overline{v \varphi}$ for some $v \in S$ and

$$
v \pi=v \varphi \pi^{\prime}=\overline{v \varphi} \pi^{\prime}=u \pi^{\prime}=g .
$$

Hence $v \in S_{g}$ and so $S_{g}^{\prime} \subseteq \overline{S_{g} \varphi}$.

Conversely, let $v \in S_{g}$. Then $\overline{v \varphi} \in \overline{S \varphi}=S^{\prime}$ and

$$
\overline{v \varphi} \pi^{\prime}=v \varphi \pi^{\prime}=v \pi=g,
$$

hence $\overline{v \varphi} \in S_{g}^{\prime}$ and so (3.4) holds.

Since

$$
\left(S^{\prime}\right)^{-1}=(\overline{S \varphi})^{-1}=\overline{(S \varphi)^{-1}}=\overline{S^{-1} \varphi}=\overline{S \varphi}=S^{\prime},
$$

it follows from (3.4) that $S^{\prime}$ is a section for $\pi^{\prime}$. Moreover, (S1) is inherited by $S^{\prime}$ from $S$ by Proposition 2.1 (ii) and Theorem 2.5. Finally, for all $g, h \in G$, we get

$$
\begin{aligned}
S_{g h}^{\prime} & =\overline{S_{g h} \varphi} \subseteq \overline{\left(\overline{S_{g} S_{h}}\right) \varphi}=\overline{\left(S_{g} S_{h}\right) \varphi} \\
& =\overline{\left(S_{g} \varphi\right)\left(S_{h} \varphi\right)}=\overline{\left(\overline{S_{g} \varphi}\right)\left(\overline{S_{h} \varphi}\right)}=\overline{S_{g}^{\prime} S_{h}^{\prime}},
\end{aligned}
$$

hence (S2) holds for $S^{\prime}$ and so $S^{\prime}$ is a Stallings section for $\pi^{\prime}$. By symmetry, we get the required equivalence.

Proposition 3.2. Free groups of finite rank and finite groups have Stallings sections.

Proof. Let $A$ be a finite set and consider the canonical m-epi $\theta: \widetilde{A}^{*} \rightarrow F_{A}$. Let

$$
S=R_{A}=\widetilde{\widetilde{A}^{*}},
$$

which is rational by Theorem 2.5. Since $S_{g}=\{\bar{g}\}$ for every $g \in F_{A}$, it is immediate that $S$ is a Stallings section for $\theta$. 
Assume now that $G$ is finite and $\pi: \widetilde{A}^{*} \rightarrow G$ is an m-epi. We show that $S=R_{A}$ is a Stallings section for $\pi$. For every $g \in G$, we have $S_{g}=g \pi^{-1} \cap R_{A}=\overline{g \pi^{-1}}$. Since both $g \pi^{-1}$ and $R_{A}$ are effectively constructible rational languages, so is their intersection and so (S1) holds. Finally, let $u \in S_{g h}$ and take $v \in S_{h}$. Then

$$
\left(u v^{-1}\right) \pi=g h h^{-1}=g
$$

and so $\overline{u v^{-1}} \in \overline{g \pi^{-1}}=S_{g}$. Hence

$$
u=\overline{u v^{-1} v}=\overline{\overline{u v^{-1}} v} \in \overline{S_{g} S_{h}}
$$

and (S2) holds as well. Therefore $R_{A}$ is a Stallings section for $\pi$.

Given an m-epi $\pi: \widetilde{A}^{*} \rightarrow G$ and $H \leqslant G$, we define the Schreier automaton $\Gamma(G, H, \pi)$ to be the $\widetilde{A}$-automaton having:

- the right cosets $H g(g \in G)$ as vertices,

- $H$ as the basepoint,

- edges $H g \stackrel{a}{\rightarrow} H g(a \pi)$ for all $g \in G$ and $a \in \widetilde{A}$.

It is immediate that $\Gamma(G, H, \pi)$ is always an inverse $\widetilde{A}$-automaton, but it is infinite unless $H$ has finite index in $G$. Moreover, $L(\Gamma(G, H, \pi))=H \pi^{-1}$.

We will prove that $\Gamma(G, H, \pi) \sqcap S$ is an effectively constructible finite inverse automaton when $S$ is a Stallings section for $\pi$. The following lemmas pave the way for the construction of $\Gamma(G, H, \pi) \sqcap S$ :

Lemma 3.3. Let $\pi: \widetilde{A}^{*} \rightarrow G$ be an m-epi. Let As be a trim $\widetilde{A}$-automaton and let

$$
p \stackrel{a}{\longrightarrow} q
$$

be an edge of $\mathcal{A}$ for some $a \in \widetilde{A}$. Let $\mathfrak{B}$ be obtained by adding the edge

$$
q \stackrel{a^{-1}}{\longrightarrow} p
$$

to A. Then $(L(\mathscr{B})) \pi \subseteq\langle(L(\mathcal{A})) \pi\rangle$.

Proof. Write $\mathcal{A}=\left(Q, q_{0}, T, E\right)$. We can factor any $u \in L(\mathscr{B})$ as

$$
u=u_{0} a^{-1} u_{1} \cdots a^{-1} u_{n},
$$

where $a^{-1}$ labels each visit to the new edge. We show that $u \pi \in\langle(L(\mathcal{A})) \pi\rangle$ by induction on $n$. The case $n=0$ being trivial, assume that $n \geq 1$ and the claim holds for $n-1$. Writing $v=u_{0} a^{-1} u_{1} \cdots a^{-1} u_{n-1}$, we have a path in $\mathscr{B}$ of the form

$$
q_{0} \stackrel{v}{\longrightarrow} q \stackrel{a^{-1}}{\longrightarrow} p \stackrel{u_{n}}{\longrightarrow} t \in T .
$$


Since $\mathcal{A}$ is trim, we also have a path

$$
q_{0} \stackrel{w}{\rightarrow} p \stackrel{a}{\rightarrow} q \stackrel{z}{\rightarrow} t^{\prime} \in T
$$

in $\mathcal{A}$. By the induction hypothesis, we get $(v z) \pi \in\langle(L(\mathcal{A})) \pi\rangle$ and so

$$
u \pi=\left(v a^{-1} u_{n}\right) \pi=\left((v z)\left(z^{-1} a^{-1} w^{-1}\right)\left(w u_{n}\right)\right) \pi \in\langle(L(\mathcal{A})) \pi\rangle
$$

as claimed.

Lemma 3.4. Let $\pi: \widetilde{A}^{*} \rightarrow G$ be an $m$-epi, and let $\mathcal{A}=\left(Q, q_{0}, T, E\right)$ be a trim $\widetilde{A}$-automaton and let $\mathscr{B}$ be obtained by identifying $q_{0}$ with some $t \in T$. Then $(L(\mathscr{B})) \pi \subseteq\langle(L(\mathcal{A})) \pi\rangle$.

Proof. Let $u \in L(\mathscr{B})$. We can factor it as $u=u_{1} \cdots u_{n}$, where $p_{i} \stackrel{u_{i}}{\longrightarrow} q_{i}$ is a path in $\mathcal{A}$ with $p_{i}, q_{i} \in\left\{q_{0}, t\right\}(i=1, \ldots, n)$. In any case, there exist paths

$$
q_{0} \stackrel{v_{i}}{\longrightarrow} p_{i}, \quad q_{i} \stackrel{w_{i}}{\longrightarrow} t \in T
$$

in $\mathcal{A}$ with $v_{i}, w_{i} \in L(\mathcal{A}) \cup\{1\}$. Since $v_{i} u_{i} w_{i} \in L(\mathcal{A})$, we get

$$
u_{i} \pi=\left(v_{i}^{-1}\left(v_{i} u_{i} w_{i}\right) w_{i}^{-1}\right) \pi \in\langle(L(\mathcal{A})) \pi\rangle
$$

for every $i$ and so $u \pi \in\langle(L(\mathcal{A})) \pi\rangle$ as well.

Lemma 3.5. Let $\pi: \widetilde{A}^{*} \rightarrow G$ be an m-epi. Let $\mathcal{A}$ be an involutive $\widetilde{A}$-automaton and let $p \stackrel{w}{\rightarrow} q$ be a path in $\mathcal{A}$ with $w \pi=1$. Let $\mathbb{B}$ be obtained by identifying the vertices $p$ and $q$. Then $L(\mathcal{A}) \subseteq L(\mathscr{B})$ and $(L(\mathscr{B})) \pi=(L(\mathcal{A})) \pi$.

Proof. The first inclusion is clear. Since $\mathcal{A}$ is involutive, we have also a path

$$
q \stackrel{w^{-1}}{\longrightarrow} p
$$

in $\mathcal{A}$ and $w^{-1} \pi=1$. Clearly, every $u \in L(\mathscr{B})$ can be lifted to some $v \in L(\mathcal{A})$ by inserting finitely many occurrences of the words $w, w^{-1}$, that is, we can get factorizations

$$
u=u_{0} u_{1} \cdots u_{n} \in L(\mathscr{B}), \quad v=u_{0} w^{\varepsilon_{1}} u_{1} \cdots w^{\varepsilon_{n}} u_{n} \in L(\mathcal{A})
$$

with $\varepsilon_{1}, \ldots, \varepsilon_{n} \in\{-1,1\}$. Since $u \pi=v \pi$, it follows that $(L(\mathscr{B})) \pi \subseteq(L(\mathcal{A})) \pi$. The opposite inclusion holds trivially.

Since $\left(a a^{-1}\right) \pi=1$ for every $a \in \widetilde{A}$, this same argument proves:

Lemma 3.6. Let $\pi: \widetilde{A}^{*} \rightarrow G$ be an m-epi. Let A be a finite involutive $\widetilde{A}$-automaton and let $\mathscr{B}$ be obtained by successively folding pairs of edges in $\mathcal{A}$. Then one has $L(\mathcal{A}) \subseteq L(\mathscr{B})$ and $(L(\mathscr{B})) \pi=(L(\mathcal{A})) \pi$. 
The next lemma reveals how the automaton $\Gamma(G, H, \pi) \sqcap S$ can be recognized.

Lemma 3.7. Let $S \subseteq R_{A}$ be a Stallings section for the m-epi $\pi: \widetilde{A}^{*} \rightarrow G$, and let $H \leqslant$ f.g. G. Let $A$ be a finite inverse $\widetilde{A}$-automaton with a basepoint such that

$$
S_{H} \subseteq L(\mathcal{A}) \subseteq H \pi^{-1}
$$

and

$$
\text { there is no path } p \stackrel{w}{\rightarrow} q \text { in } \mathcal{A} \text { with } p \neq q \text { and } w \pi=1 .
$$

Then $\Gamma(G, H, \pi) \sqcap S \cong \mathcal{A} \sqcap S$.

Proof. Since $\mathcal{A}$ and $\Gamma=\Gamma(G, H, \pi)$ are both inverse automata with a basepoint, and $L(\mathcal{A}) \subseteq H \pi^{-1}=L(\Gamma)$, it follows from Proposition 2.4 that there exists a morphism $\varphi: \mathcal{A} \rightarrow \Gamma$. Suppose that $p \varphi=q \varphi$ for some vertices $p, q$ in $\mathcal{A}$. Take geodesics

$$
q_{0} \stackrel{u}{\rightarrow} p, \quad q_{0} \stackrel{v}{\rightarrow} q
$$

in $\mathcal{A}$, where $q_{0}$ denotes the basepoint. Since $p \varphi=q \varphi$, we have

$$
u v^{-1} \in L(\Gamma)=H \pi^{-1} .
$$

Let $s_{0} \in S_{\left(u v^{-1}\right) \pi} \subseteq S_{H}$. Then $s_{0} \in L(\mathcal{A})$ by (3.5) and so there is a path

$$
p \stackrel{u^{-1} s_{0} v}{\longrightarrow} q
$$

in $\mathcal{A}$. Since $\left(u^{-1} s_{0} v\right) \pi=\left(u^{-1} u v^{-1} v\right) \pi=1$, it follows from (3.6) that $p=q$. Thus $\varphi$ is injective.

It is immediate that $\varphi$ restricts to an injective morphism $\varphi^{\prime}: \mathcal{A} \sqcap S \rightarrow \Gamma \sqcap S$. It remains to show that every edge of $\Gamma \sqcap S$ is induced by some edge of $\mathcal{A} \sqcap S$. Assume that $H \stackrel{s}{\rightarrow} H$ is a (successful) path in $\Gamma$ with $s \in S$. By (3.5), we have $s \in L(\mathcal{A})$ and the path $q_{0} \stackrel{s}{\rightarrow} q_{0}$ is mapped by $\varphi^{\prime}$ onto $H \stackrel{s}{\rightarrow} H$. Since every edge of $\Gamma \sqcap S$ occurs in some path $H \stackrel{s}{\rightarrow} H$, it follows that $\varphi^{\prime}$ is an isomorphism.

Lemma 3.8. Let $S \subseteq R_{A}$ be a Stallings section for the m-epi $\pi: \widetilde{A}^{*} \rightarrow G$, and let $H \leqslant$ f.g. G. Let $\mathcal{A}$ be a finite inverse $\widetilde{A}$-automaton with a basepoint such that $S_{H} \subseteq L(\mathcal{A}) \subseteq H \pi^{-1}$. It is decidable, given two distinct vertices $p$ and $q$ of $\mathcal{A}$, whether or not there is some path $p \stackrel{w}{\rightarrow} q$ in $\mathcal{A}$ with $w \pi=1$.

Proof. Let $p, q$ be distinct vertices of $\mathcal{A}$ and let $q_{0}$ denote its basepoint. Take geodesics $q_{0} \stackrel{u}{\rightarrow} p$ and $q_{0} \stackrel{v}{\rightarrow} q$, and let $s \in S_{\left(u v^{-1}\right) \pi}$. We claim that there is a path $p \stackrel{w}{\rightarrow} q$ in $\mathcal{A}$ with $w \pi=1$ if and only if $s \in L(\mathcal{A})$.

Indeed, assume that $p \stackrel{w}{\rightarrow} q$ is such a path. Then $u w v^{-1} \in L(\mathcal{A})$ and so we have $s \pi=\left(u v^{-1}\right) \pi=\left(u w v^{-1}\right) \pi \in H$. Thus $s \in S_{H} \subseteq L(\mathcal{A})$. 
Conversely, assume that $s \in L(\mathcal{A})$. Then there is a path $p \stackrel{u^{-1} s v}{\longrightarrow} q$ in $\mathcal{A}$. Since $\left(u^{-1} s v\right) \pi=\left(u^{-1} u v^{-1} v\right) \pi=1$, the lemma is proved.

Theorem 3.9. Let $S \subseteq R_{A}$ be a Stallings section for the m-epi $\pi: \widetilde{A}^{*} \rightarrow G$ and let $H \leqslant f . g$. G. Then $\Gamma(G, H, \pi) \sqcap S$ is an effectively constructible finite inverse $\widetilde{A}$-automaton with a basepoint such that

$$
S_{H} \subseteq L(\Gamma(G, H, \pi) \sqcap S) \subseteq H \pi^{-1} .
$$

Proof. Assume that $H=\left\langle h_{1}, \ldots, h_{m}\right\rangle$. For $i=1, \ldots, m$, let $\mathcal{A}_{i}=\left(Q_{i}, q_{i}, t_{i}, E_{i}\right)$ be a finite trim $\widetilde{A}$-automaton with a single initial and a single terminal vertex satisfying

$$
S_{h_{i}} \subseteq \overline{L\left(\mathcal{A}_{i}\right)} \subseteq h_{i} \pi^{-1}
$$

(in the next section we shall discuss how to define such an automaton with the lowest possible complexity). Let $\mathscr{B}_{0}$ be the $\widetilde{A}$-automaton obtained by taking the disjoint union of the $\mathcal{A}_{i}$ and then identifying all the $q_{i}$ into a single initial vertex $q_{0}$.

Suppose that $q_{i} \stackrel{u}{\rightarrow} q_{i}$ is a path in $\mathcal{A}_{i}$. Take $v \in L\left(\mathcal{A}_{i}\right)$. Then

$$
u v \in L\left(\mathcal{A}_{i}\right) \subseteq h_{i} \pi^{-1}
$$

and so $u \pi=\left(u v v^{-1}\right) \pi=h_{i} h_{i}^{-1}=1$. It follows easily that

$$
\left(L\left(B_{0}\right)\right) \pi \subseteq\left(S_{h_{1}} \cup \cdots \cup S_{h_{m}}\right) \pi \subseteq H .
$$

Let $\mathscr{B}_{1}$ be the finite, trim, involutive $\widetilde{A}$-automaton obtained from $\mathscr{B}_{0}$ by adjoining edges $\left(q, a^{-1}, p\right)$ for all edges $(p, a, q)$ in $\mathscr{B}_{0}(a \in \widetilde{A})$. It follows from Lemma 3.3 that

$$
\left(L\left(\mathscr{B}_{1}\right)\right) \pi \subseteq\left\langle\left(L\left(\mathscr{B}_{0}\right)\right) \pi\right\rangle \subseteq H .
$$

Next, let $\mathscr{B}_{2}$ be the $\widetilde{A}$-automaton obtained from $\mathscr{B}_{1}$ by identifying all terminal vertices with the initial vertex $q_{0}$. By Lemma 3.4 , we get

$$
\left(L\left(\mathscr{B}_{2}\right)\right) \pi \subseteq\left\langle\left(L\left(\mathscr{B}_{1}\right)\right) \pi\right\rangle \subseteq H .
$$

Finally, let $\mathscr{B}_{3}$ be the finite, inverse $\widetilde{A}$-automaton with a basepoint obtained by complete folding of $\mathscr{B}_{2}$. By Lemma 3.6, we have $\left(L\left(\mathscr{B}_{3}\right)\right) \pi=\left(L\left(\mathscr{B}_{2}\right)\right) \pi \subseteq H$ and so $L\left(\mathscr{B}_{3}\right) \subseteq H \pi^{-1}$. Moreover,

$$
S_{h_{1}} \cup \cdots \cup S_{h_{m}} \subseteq \overline{L\left(\mathcal{A}_{1}\right)} \cup \cdots \cup \overline{L\left(\mathcal{A}_{m}\right)} \subseteq \overline{L\left(\mathscr{B}_{0}\right)} \subseteq \overline{L\left(\mathscr{B}_{3}\right)}
$$

and $S^{-1}=S$ yield

$$
\overline{\left(S_{h_{1}} \cup \cdots \cup S_{h_{m}} \cup S_{h_{1}^{-1}} \cup \cdots \cup S_{h_{m}^{-1}}\right)^{*}} \subseteq \overline{L\left(\mathscr{B}_{3}\right)}
$$

since $\mathscr{B}_{3}$ is involutive and has a basepoint, and therefore

$$
\overline{\left(S_{h_{1}} \cup \cdots \cup S_{h_{m}} \cup S_{h_{1}^{-1}} \cup \cdots \cup S_{h_{m}^{-1}}\right)^{*}} \subseteq L\left(\mathscr{B}_{3}\right)
$$


since $\mathscr{B}_{3}$ is inverse (the language of an inverse automaton is closed under reduction since a word $a a^{-1}$ must label only loops). In view of (3.2), it follows that $S_{h} \subseteq L\left(\mathscr{B}_{3}\right)$ for every $h \in H$ and so $S_{H} \subseteq L\left(\mathscr{B}_{3}\right)$. Therefore, (3.5) holds for $\mathscr{B}_{3}$.

However, (3.6) may not hold. Assume that the vertex set $Q^{\prime}$ of $\mathscr{B}_{3}$ is totally ordered. By Lemma 3.8, we can decide if that happens, and find all concrete instances

$J=\left\{(p, q) \in Q^{\prime} \times Q^{\prime} \mid p<q\right.$, there is some path $p \stackrel{w}{\rightarrow} q$ in $\mathscr{B}_{3}$ with $\left.w \pi=1\right\}$.

Let $\mathscr{B}_{4}$ be the finite inverse $\widetilde{A}$-automaton with a basepoint obtained by identifying all pairs of vertices in $J$ followed by complete folding. Since the existence of a path with label in $1 \pi^{-1}$ is preserved through the identification process, it follows from Lemmas 3.5 and 3.6 that $\mathscr{B}_{4}$ still satisfies (3.5).

Suppose that there exists a path

$$
p^{\prime} \stackrel{w^{\prime}}{\rightarrow} q^{\prime}
$$

in $\mathscr{B}_{4}$ with $p^{\prime} \neq q^{\prime}$ and $w^{\prime} \pi=1$. We can lift $p^{\prime}$ and $q^{\prime}$ to vertices $p$ and $q$ in $\mathscr{B}_{3}$, respectively. It is straightforward to check that the path $(*)$ can be lifted to a path $p \stackrel{w}{\rightarrow} q$ in $\mathscr{B}_{3}$ by successively inserting in $w^{\prime}$ factors of the form:

- $a a^{-1}(a \in \widetilde{A})$ (undoing the folding operations),

- $z \in 1 \pi^{-1}$ (undoing the identification arising from $r \stackrel{z}{\rightarrow} s$ ).

Since $w^{\prime} \pi=w \pi$, it follows that either $(p, q) \in J$ or $(q, p) \in J$, and so $p^{\prime}=q^{\prime}$, a contradiction. Therefore $\mathscr{B}_{4}$ satisfies (3.6). Now the theorem follows from Proposition 2.3 and Lemma 3.7.

We call $\Gamma(G, H, \pi) \sqcap S$ the Stallings automaton of $H$ (for a given Stallings section $S)$. Note that $\Gamma\left(F_{A}, H, \theta\right) \sqcap R_{A}$ is the classical Stallings automaton of $H \leq_{f . g .} F_{A}$ when we take $R_{A}$ as Stallings section (for the canonical m-epi $\theta$ ).

Stallings automata provide a natural decision procedure for the generalized word problem:

Corollary 3.10. Let $S \subseteq R_{A}$ be a Stallings section for the $m$-epi $\pi: \widetilde{A}^{*} \rightarrow G$ and let $H \leqslant$ f.g. $G$. Then the following conditions are equivalent for every $g \in G$ :

(a) $g \in H$,

(b) $S_{g} \subseteq L(\Gamma(G, H, \pi) \sqcap S)$,

(c) $S_{g} \cap L(\Gamma(G, H, \pi) \sqcap S) \neq \varnothing$.

Furthermore, the generalized word problem is decidable for $G$. 
Proof. For (a) $\Rightarrow$ (b), observe that if $g \in H$, then $S_{g} \subseteq S_{H} \subseteq L(\Gamma(G, H, \pi) \sqcap S)$ by Theorem 3.9. The implication (b) $\Rightarrow$ (c) is immediate since $S_{g} \neq \emptyset$ due to $S$ being a section. And (c) $\Rightarrow$ (a) is true because

$$
S_{g} \cap L(\Gamma(G, H, \pi) \sqcap S) \subseteq g \pi^{-1} \cap H \pi^{-1} .
$$

Finally, decidability follows from (S1) and Theorem 3.9.

We can also prove the following generalization of Theorem 2.5:

Theorem 3.11. Let $S \subseteq R_{A}$ be a Stallings section for the m-epi $\pi: \widetilde{A}^{*} \rightarrow G$ and let $L \subseteq \widetilde{A}^{*}$ be rational. Then $S_{L \pi}$ is an effectively constructible rational language.

Proof. Let $\varphi: \widetilde{A}^{*} \rightarrow$ Rat $\widetilde{A}^{*}$ be the rational substitution defined by $a \varphi=S_{a \pi}$, for $a \in \widetilde{A}$ (note that $1 \varphi=\{1\}$ and, for $u=a_{1} \cdots a_{n}\left(a_{i} \in \widetilde{A}\right), u \varphi$ is not $S_{u \pi}$ but just $\left.S_{a_{1} \pi} \cdots S_{a_{n} \pi}\right)$. We claim that

$$
S_{u \pi}=S \cap \overline{u \varphi}
$$

holds for every $u \in L \backslash\{1\}$. Let $u=a_{1} \cdots a_{n} \in L\left(a_{i} \in \widetilde{A}\right)$. Then by (3.2) we get

$$
S_{u \pi}=S_{\left(a_{1} \pi\right) \cdots\left(a_{n} \pi\right)} \subseteq \overline{S_{a_{1} \pi} \cdots S_{a_{n} \pi}}=\overline{\left(a_{1} \varphi\right) \cdots\left(a_{n} \varphi\right)}=\overline{u \varphi}
$$

and so $S_{u \pi} \subseteq S \cap \overline{u \varphi}$.

Since $a \varphi \pi=S_{a \pi} \pi=a \pi$ holds for every $a \in \widetilde{A}$, the inclusion $S \cap \overline{u \varphi} \subseteq S_{u \pi}$ follows from $\overline{u \varphi} \pi=u \varphi \pi=u \pi$. Therefore (3.9) holds.

Now it becomes clear that

$$
S_{L \pi}=S \cap\left(\bigcup_{u \in L} \overline{u \varphi}\right)=S \cap \overline{L \varphi}
$$

if $1 \notin L$ and

$$
S_{L \pi}=(S \cap \overline{L \varphi}) \cup S_{1}
$$

if $1 \in L$. And $L \varphi$ is an effectively constructible rational language by (S1) and Proposition 2.1 (ii), and so is $\overline{L \varphi}$ by Theorem 2.5. Since $S, S_{1}$ are rational, it follows from Proposition 2.1 (i) that $S_{L \pi}$ is rational and effectively constructible.

A natural question to ask at this stage is if we can identify a Stallings automaton for a given Stallings section $S$. In the classical case of a free group $F_{A}$ with $S=R_{A}$ this is an elementary thing to do: in this case, an $\widetilde{A}$-automaton $\mathcal{A}$ is of the form $\Gamma\left(F_{A}, H, \pi\right) \sqcap R_{A}=\wp_{H}$ for some $H \leqslant{ }_{f . g} . F_{A}$ if and only if $\mathcal{A}$ is inverse, has a basepoint, and has no vertex of outdegree one except possibly the basepoint.

Proposition 3.12. Let $S \subseteq R_{A}$ be a Stallings section for an m-epi $\pi: \widetilde{A}^{*} \rightarrow G$. It is decidable, given a finite $\widetilde{\widetilde{A}}$-automaton $\mathcal{A}$, whether or not $\mathcal{A} \cong \Gamma(G, H, \pi) \sqcap S$ for some $H \leqslant f . g$. $G$. 
Proof. We may assume that $\mathcal{A}$ is inverse and has a basepoint. Write

$$
\mathcal{A}=\left(Q, q_{0}, q_{0}, E\right) .
$$

The equality $\mathcal{A}=\mathcal{A} \sqcap S$ is an obvious necessary condition, decidable by Lemma 2.3. Thus we may assume that $\mathcal{A}=\mathcal{A} \sqcap S$ (in particular, $\mathcal{A}$ is trim).

Since $S \subseteq R_{A}$ and $\mathcal{A}$ is trim, it follows that only the basepoint may have outdegree 1 , and so

$$
\mathcal{A} \cong \mathcal{S}(K) \cong \Gamma\left(F_{A}, K, \theta\right) \sqcap R_{A}
$$

for some $K \leqslant_{f . g .} F_{A}$, see [1, Proposition 2.12]: the standard algorithm [1, Proposition 2.6] actually computes a finite subset $X \subseteq R_{A}$ projecting onto a basis $X \theta$ of $K$. Let $K^{\prime}=\langle X \pi\rangle \leqslant f . g$. $G$. We claim that $\mathcal{A} \cong \Gamma(G, H, \pi) \sqcap S$ for some $H \leqslant f . g . G$ if and only if $\mathcal{A} \cong \Gamma\left(G, K^{\prime}, \pi\right) \sqcap S$, a decidable condition in view of Theorem 3.9.

The converse implication being trivial, assume that $\mathcal{A}=\Gamma(G, H, \pi) \sqcap S$ for some $H \leqslant f . g$. $G$. Since words of $1 \pi^{-1}$ can only label loops in $\Gamma(G, H, \pi)$, it follows from Lemma 3.7 that we only need to show that

$$
S_{K^{\prime}} \subseteq L(\mathcal{A}) \subseteq K^{\prime} \pi^{-1} .
$$

Since $\mathcal{A} \cong \Gamma\left(F_{A}, K, \theta\right) \sqcap R_{A}$, it follows from Theorem 3.9 that

$$
X \subseteq R_{A} \cap K \theta^{-1} \subseteq L(\mathcal{A}) \subseteq K \theta^{-1} .
$$

Since $K \theta^{-1} \subseteq K^{\prime} \pi^{-1}$, we get

$$
L(\mathcal{A}) \subseteq K^{\prime} \pi^{-1} .
$$

Finally, $X \subseteq L(\mathcal{A}) \subseteq H \pi^{-1}$ yields $X \pi \subseteq H$ and so $K^{\prime} \leqslant H$. Hence,

$$
S_{K^{\prime}} \subseteq S_{H} \subseteq L(\mathcal{A})
$$

by (3.7) and so (3.10) holds. Thus $\mathcal{A} \cong \Gamma\left(G, K^{\prime}, \pi\right) \sqcap S$ and we are done.

\section{Complexity}

In this section we discuss, for a given Stallings section, an efficient way (from the viewpoint of complexity) of constructing the automata $\mathcal{A}_{i}$ in the proof of Theorem 3.9 and compute an upper bound for the complexity of the construction of the Stallings automata $\Gamma(G, H, \pi) \sqcap S$.

We say that an $\widetilde{A}$-automaton is uniterminal if it has a single terminal vertex. It is easy to see that there exist rational languages which fail to be recognized by any uniterminal automaton (e.g. $R_{A}$, since regular languages recognizable by uniterminal automata and containing the empty word must have a basepoint and so they are submonoids). However, we can prove the following lemma. 
Lemma 4.1. Let $S \subseteq R_{A}$ be a Stallings section for the $m$-epi $\pi: \widetilde{A}^{*} \rightarrow G$ and let $g \in G$. Then there exists a finite trim uniterminal $\widetilde{A}$-automaton $\mathcal{C}_{g}$ satisfying

$$
S_{g} \subseteq \overline{L\left(\mathcal{C}_{g}\right)} \subseteq g \pi^{-1} .
$$

Proof. Let $\mathcal{C}=(Q, i, T, E)$ be the minimum automaton of $S_{g}$ (or any other finite trim automaton with a single initial vertex recognizing $S_{g}$ ) and let $\mathcal{C}_{g}$ be obtained by identifying all the terminal vertices of $\mathcal{C}$. Clearly, $\ell_{g}$ is a finite trim uniterminal automaton and $S_{g}=L(\mathcal{C}) \subseteq L\left(\mathcal{C}_{g}\right)$ yields $S_{g}=\overline{S_{g}} \subseteq \overline{L\left(\mathcal{C}_{g}\right)}$. It remains to prove that $\left(L\left(\mathcal{C}_{g}\right)\right) \pi=g$.

Let $u \in L\left(\varphi_{g}\right)$. Then there exists a factorization $u=u_{0} u_{1} \cdots u_{k}$ such that

$$
i \stackrel{u_{0}}{\longrightarrow} t_{0}, s_{1} \stackrel{u_{1}}{\longrightarrow} t_{1}, \ldots, s_{k} \stackrel{u_{k}}{\longrightarrow} t_{k}
$$

are paths in $\mathcal{C}$ with $s_{j}, t_{j} \in T$. Take a path $i \stackrel{v_{j}}{\longrightarrow} s_{j}$ in $\mathcal{C}$, for $j=1, \ldots, k$. Then $v_{j}, v_{j} u_{j} \in L(\mathcal{C})$ and so $v_{j} \pi=\left(v_{j} u_{j}\right) \pi=g$. Hence,

$$
u_{j} \pi=\left(v_{j}^{-1} v_{j} u_{j}\right) \pi=g^{-1} g=1
$$

and so

$$
u \pi=\left(u_{0} u_{1} \cdots u_{k}\right) \pi=u_{0} \pi=g
$$

since $u_{0} \in L(\mathcal{C})=S_{g}$. Thus, $\left(L\left(\mathcal{C}_{g}\right)\right) \pi=g$ and so

$$
\overline{L\left(\mathcal{C}_{g}\right)} \subseteq g \pi^{-1}
$$

as required.

Next we introduce a multiplication of (finite trim) uniterminal automata: given (finite trim) uniterminal $\widetilde{A}$-automata $\mathcal{A}=(Q, i, t, E)$ and $\mathcal{A}^{\prime}=\left(Q^{\prime}, i^{\prime}, t^{\prime}, E^{\prime}\right)$, let $\mathcal{A} * \mathcal{A}^{\prime}=\left(Q^{\prime \prime}, i, t^{\prime}, E^{\prime \prime}\right)$ be the (finite trim) uniterminal $\widetilde{A}$-automaton obtained by taking the disjoint union of the underlying graphs of $\mathcal{A}$ and $\mathcal{A}^{\prime}$ and identifying $t$ with $i^{\prime}$.

Lemma 4.2. Let $S \subseteq R_{A}$ be a Stallings section for the m-epi $\pi: \widetilde{A}^{*} \rightarrow G$, and let $g, g^{\prime} \in G$. Let $\mathcal{A}$ and $\mathcal{A}^{\prime}$ be finite trim uniterminal $\widetilde{A}$-automata satisfying

$$
S_{g} \subseteq \overline{L(\mathcal{A})} \subseteq g \pi^{-1}, \quad S_{g^{\prime}} \subseteq \overline{L\left(\mathcal{A}^{\prime}\right)} \subseteq g^{\prime} \pi^{-1} .
$$

Then

$$
S_{g g^{\prime}} \subseteq \overline{L\left(\mathcal{A} * \mathcal{A}^{\prime}\right)} \subseteq\left(g g^{\prime}\right) \pi^{-1} .
$$

Proof. Since $L(\mathcal{A}) L\left(\mathcal{A}^{\prime}\right) \subseteq L\left(\mathcal{A} * \mathcal{A}^{\prime}\right)$, we get in view of (S2)

$$
S_{g g^{\prime}} \subseteq \overline{S_{g} S_{g^{\prime}}} \subseteq \overline{L(\mathcal{A}) L\left(\mathcal{A}^{\prime}\right)} \subseteq \overline{L\left(\mathcal{A} * \mathcal{A}^{\prime}\right)}
$$


Now let $u \in L\left(\mathcal{A} * \mathcal{A}^{\prime}\right)$. Then $u$ labels a path in $\mathcal{A} * \mathcal{A}^{\prime}$ of the form

$$
i \stackrel{u_{0}}{\longrightarrow} p \stackrel{u_{1}}{\longrightarrow} p \stackrel{u_{2}}{\longrightarrow} \cdots \stackrel{u_{k-1}}{\longrightarrow} p \stackrel{u_{k}}{\longrightarrow} t^{\prime},
$$

where we emphasize all the occurrences of the vertex $p$ obtained through the identification of $t$ and $i^{\prime}$. Now it is easy to see that there exist paths

$$
i \stackrel{u_{0}}{\longrightarrow} t \quad \text { and } \quad i^{\prime} \stackrel{u_{k}}{\longrightarrow} t^{\prime}
$$

in $\mathcal{A}$ and $\mathcal{A}^{\prime}$, respectively. Moreover, for each $j=1, \ldots, k-1$, there exists either a path

$$
t \stackrel{u_{j}}{\longrightarrow} t
$$

in $\mathcal{A}$ or a path

$$
i^{\prime} \stackrel{u_{j}}{\longrightarrow} i^{\prime}
$$

in $\mathcal{A}^{\prime}$. Now, in view of $(L(\mathcal{A})) \pi=g$ and $\left(L\left(\mathcal{A}^{\prime}\right)\right) \pi=g^{\prime}$, we can use the same argument as in the proof of Lemma 4.1 to show that $u_{j} \pi=1$ for $j=1, \ldots, k-1$. Hence $u \pi=\left(u_{0} u_{1} \cdots u_{k}\right) \pi=\left(u_{0} u_{k}\right) \pi=g g^{\prime}$ and so $\overline{L\left(\mathcal{A} * \mathcal{A}^{\prime}\right)} \subseteq\left(g g^{\prime}\right) \pi^{-1}$ as required.

In view of the preceding two lemmas, we can now set an algorithm to construct the automata $\mathcal{A}_{i}$ in the proof of Theorem 3.9. All we need for a start are the minimum automata of $S_{a \pi}$ for each $a \in A$ (or any other finite trim automaton with a single initial vertex recognizing $S_{a \pi}$; this can be effectively constructed by (S1)). Following the argument in the proof of Lemma 4.1, we may identify all the terminal vertices to get finite trim uniterminal $\widetilde{A}$-automata $C_{a \pi}$ satisfying

$$
S_{a \pi} \subseteq \overline{L\left(\mathcal{C}_{a \pi}\right)} \subseteq a \pi \pi^{-1} .
$$

Note that, since $S^{-1}=S$, we get finite trim uniterminal $\widetilde{A}$-automata $\bigodot_{a^{-1} \pi}$ satisfying

$$
S_{a^{-1} \pi} \subseteq \overline{L\left(\bigodot_{a^{-1} \pi}\right)} \subseteq a^{-1} \pi \pi^{-1}
$$

by exchanging the initial and the terminal vertices in $\mathcal{C}_{a \pi}$ and replacing each edge

$$
p \stackrel{b}{\longrightarrow} q
$$

by an edge

$$
q \stackrel{b^{-1}}{\longrightarrow} p .
$$

Now, given an element $h_{i} \in G$, we may represent it by some reduced word $a_{1} \cdots a_{n}\left(a_{i} \in \widetilde{A}\right)$, and may compute

$$
\mathcal{A}_{i}=\left(\left(\cdots\left(\mathcal{C}_{a_{1} \pi} * \boldsymbol{C}_{a_{2} \pi}\right) * \boldsymbol{C}_{a_{3} \pi}\right) * \cdots\right) * \mathcal{C}_{a_{n} \pi} .
$$


By Lemma $4.2, \mathcal{A}_{i}$ is a finite trim uniterminal $\widetilde{A}$-automaton satisfying

$$
S_{h_{i}} \subseteq \overline{L\left(\mathcal{A}_{i}\right)} \subseteq h_{i} \pi^{-1}
$$

What is the maximum size of $\mathcal{A}_{i}$ relatively to $\left|h_{i}\right|$ ? What is the time complexity of the algorithm for its construction? Note that we start with only finitely many "atomic" automata $\bigodot_{a \pi}(a \in A)$. Hence the number of vertices (edges) in $\mathcal{A}_{i}$ is a bounded multiple of $\left|h_{i}\right|$, therefore is $O\left(\left|h_{i}\right|\right)$, and the time complexity of the construction (disjoint union followed by identification of two vertices, $\left|h_{i}\right|-1$ times) is also clearly $O\left(\left|h_{i}\right|\right)$. This is why we gave ourselves (and the reader) the trouble of constructing the $\mathcal{A}_{i}$ this way instead of just taking the minimum automaton of $S_{h_{i}}$, whatever that may be!

But what is the time complexity of the full algorithm leading to the Stallings automaton $\Gamma(G, H, \pi) \sqcap S$ ? It is also useful to discuss the complexity of the important intermediate $\mathscr{B}_{3}$ in the proof of Theorem 3.9 since $\mathscr{B}_{3}$ suffices for such applications as the generalized word problem: indeed, since $\mathcal{B}_{3}$ satisfies (3.5), we may replace $\Gamma(G, H, \pi) \sqcap S$ by $\mathcal{B}_{3}$ in Corollary 3.10 .

Let $n=\left|h_{1}\right|+\cdots+\left|h_{m}\right|$. It follows easily from our previous discussion of the time complexity of the construction of the $\mathcal{A}_{i}$ that $\mathscr{B}_{0}$ (and therefore $\mathscr{B}_{1}$ and $\mathscr{B}_{2}$ ) can be constructed in time $O(n)$. Since we get to $\mathscr{B}_{3}$ through complete folding, the complexity of constructing $\mathscr{B}_{3}$ is that of the classical Stallings construction in the free group.

The Ackermann hierarchy is a sequence $\left(A_{k}\right)_{k}$ of transformations of $\mathbb{N}$ defined by $A_{1}(n)=n$ and $A_{k}(n)=A_{k-1}^{n}$ (1) for $k>1$ (where $A_{k-1}^{n}$ denotes the $n$-fold composition of $A_{k-1}$ ). Following Nivatsch [15], we can define the Ackermann function $A: \mathbb{N} \rightarrow \mathbb{N}$ by

$$
A(n)=A_{n}(3) .
$$

The inverse Ackermann function $\alpha:[0,+\infty[\rightarrow \mathbb{N}$ is then defined by

$$
\alpha(x)=\min \{n \in \mathbb{N} \mid A(n) \geq x\} .
$$

The inverse Ackermann function grows extremely slowly.

Using a famous result of Tarjan on Union-Find [20] (see also [4]) Touikan proved in [21] that such complexity is $O(n \alpha(n))$, i.e. very close to linear. Therefore $\mathcal{B}_{3}$ can be constructed in time $O(n \alpha(n))$.

We shall now discuss the complexity of the construction of the Stallings automata:

Theorem 4.3. Let $S \subseteq R_{A}$ be a Stallings section for the $m$-epi $\pi: \widetilde{A}^{*} \rightarrow G$ and let $H=\left\langle h_{1}, \ldots, h_{m}\right\rangle \leqslant f . g$. $G$. Then $\Gamma(G, H, \pi) \sqcap S$ can be constructed in time $O\left(n^{3} \alpha(n)\right)$, where $n=\left|h_{1}\right|+\cdots+\left|h_{m}\right|$. 
Proof. We go back to the proof of Theorem 3.9, starting at $\mathcal{B}_{3}$.

The number of vertices of $\mathcal{B}_{3}$ is $O(n)$ and therefore we have $O\left(n^{2}\right)$ candidate pairs to $J$. For each one of these pairs, we must decide whether or not they belong to $J$. This involves bounding the complexity of the algorithm described in the proof of Lemma 3.8 .

Let $p, q$ be distinct vertices of $\mathcal{B}_{3}$ and let $q_{0}$ denote its basepoint. Take two geodesics $q_{0} \stackrel{u}{\rightarrow} p$ and $q_{0} \stackrel{v}{\rightarrow} q$. Clearly, $g=\left(u v^{-1}\right) \pi$ can be represented by a word of length $O(n)$. It follows from the previous discussion on the complexity of the construction of $\mathcal{A}_{i}$ that we may construct a finite trim uniterminal $\widetilde{A}$-automaton $\bigodot_{g}$ satisfying

$$
S_{g} \subseteq \overline{L\left(\digamma_{g}\right)} \subseteq g \pi^{-1}
$$

in time $O(n)$. Performing a complete folding on $\bigodot_{g}$ (in time $O(n \alpha(n))$ ), we get a finite inverse $\widetilde{A}$-automaton $\mathscr{D}_{g}$ satisfying

$$
S_{g} \subseteq L\left(\mathcal{D}_{g}\right) \subseteq g \pi^{-1}
$$

Since $S$ is a constant for our problem, we can compute an element

$$
s \in S \cap L\left(\mathscr{D}_{g}\right)=S_{g}
$$

in time $O(n)$ and check if $s \in L\left(\mathscr{B}_{3}\right)$ in time $O(n)$. Therefore, by the proof of Lemma 3.8, we can decide whether or not $(p, q) \in J$ in time $O(n \alpha(n))$. Since we had $O\left(n^{2}\right)$ candidates to consider, we may compute $J$ in time $O\left(n^{3} \alpha(n)\right)$. It is very likely that this upper bound can be improved.

Since $\mathcal{B}_{4}$ is obtained from $\mathscr{B}_{3}$ by identifying the pairs in $J$ followed by complete folding, and $\mathscr{B}_{3}$ has $O(n)$ vertices, it follows that $\mathscr{B}_{4}$ can be constructed in time $O\left(n^{3} \alpha(n)\right)$ in view of Touikan's bound.

For the last step, we must discuss the time complexity of the algorithm in the proof of Proposition 2.3. Note that $\mathscr{B}_{4}$ has $O(n)$ vertices and therefore (since the alphabet is fixed) $O(n)$ edges. Since $S$ is a constant for our problem, we can build the direct product of $\mathcal{B}_{4}$ by some deterministic automaton recognizing $S$ in time $O(n)$ and compute its trim part in time $O(n)$ (we have $O(n)$ vertices and $O(n)$ edges), and the final projection can also be performed in linear time. Therefore $\Gamma(G, H, \pi) \sqcap S$ can be constructed in time $O\left(n^{3} \alpha(n)\right)$, which means very close to cubic complexity.

We should stress that the above discussion of time complexity was performed for a fixed Stallings section of a fixed group. But the computation of a Stallings section for a (virtually free) group can be in itself a costly procedure, particularly if it is supported by Bass-Serre theory as in the present case. This will become more evident throughout the next section. 


\section{Virtually free groups}

A group is virtually free if it has a free subgroup of finite index. Some recent papers involving virtually free groups include $[5,9,10,18]$.

Next we recall the concept of graph of groups, central in Bass-Serre theory [17]. In Serre's viewpoint, a graph is a structure of the form $\Gamma=\left(V, E, \tau,{ }^{-}\right)$, where:

- $V$ is a nonempty set (vertices),

- $E$ is a set (edges),

- $\tau: E \rightarrow V$ is a mapping (target mapping),

${ }^{-}: E \rightarrow E$ is an involution without fixed points.

Concepts such as cycle, connectedness, tree or subgraph are defined in the obvious way. If $\Gamma$ is connected and $T \subseteq E$ defines a subtree of $\Gamma$ connecting all the vertices, we say that $T$ is a spanning tree of $\Gamma$.

We write $v \stackrel{e}{\rightarrow} w$ if $e \tau=w$ and $\bar{e} \tau=v$. This allows us to view $\Gamma$ as an $E$-automaton whenever convenient. Note that $v \stackrel{e}{\rightarrow} w$ if and only if $w \stackrel{\bar{e}}{\rightarrow} v$.

A (finite) graph of groups over a (finite) connected graph $\Gamma$ is a structure of the form

$$
\mathcal{E}=\left(\left(G_{v}\right)_{v \in V},\left(G_{e}\right)_{e \in E},\left(\tau_{e}\right)_{e \in E}\right),
$$

where:

- $G_{v}$ is a group for every $v \in V$ (vertex groups),

- $G_{e}$ is a group for every $e \in E$ satisfying $G_{\bar{e}}=G_{e}$ (edge groups),

- $\tau_{e}: G_{e} \rightarrow G_{e \tau}$ is a monomorphism for every edge $e \in E$ (boundary monomorphisms).

Let $P(\mathcal{G})$ denote the quotient of the free product $\left(*_{v \in V} G_{v}\right) * F_{E}$ by the normal subgroup generated by the elements of the form

$$
e\left(g \tau_{e}\right) \bar{e}\left(g \tau_{\bar{e}}\right)^{-1} \quad\left(e \in E, g \in G_{e}=G_{\bar{e}}\right) .
$$

Note that $e \bar{e}$ arises from the particular case $g=1$.

Serre presents two alternative constructions for the fundamental group of $\mathscr{E}$ :

The cycle construction. Fix $v_{0} \in V$. Let $C\left(\mathscr{G}, v_{0}\right)$ denote the set of all closed paths of the form

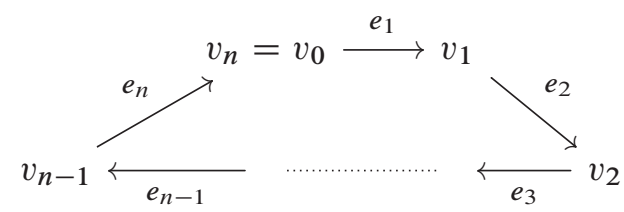

in $\Gamma$ (including the trivial path). 
The fundamental group $\pi_{1}\left(\mathcal{E}, v_{0}\right)$ of the graph of groups (5.1) with respect to $v_{0} \in V$ is the subgroup of $P(\mathscr{G})$ consisting of the following elements: for every closed path of the above form, $\pi_{1}\left(\mathcal{E}, v_{0}\right)$ contains all the elements of the form

$$
g_{0} e_{1} g_{1} \ldots e_{n} g_{n}, \quad \text { with } g_{i} \in G_{v_{i}} \text { for } i=0, \ldots, n \text {. }
$$

We shall use the notation $\left(g_{0} e_{1} g_{1} \cdots e_{n} g_{n}\right) \xi=e_{1} \cdots e_{n}$.

The spanning tree construction. Let $T$ be a spanning tree of the graph $\Gamma$. The fundamental group $\pi_{1}(\mathcal{G}, T)$ is the quotient of $P(\mathscr{G})$ by the normal subgroup generated by the edges in $T$.

Serre shows that the canonical projection $P(\mathscr{E}) \rightarrow \pi_{1}(\mathscr{E}, T)$ induces an isomorphism from $\pi_{1}\left(\mathscr{Y}, v_{0}\right)$ to $\pi_{1}(\mathscr{E}, T)$, which implies in particular that both constructions are independent from the choice of $v_{0}$ and $T$. Therefore, we can benefit from the best of both worlds: $\pi_{1}(G, T)$ provides a nice canonical generating set, while $\pi_{1}\left(G, v_{0}\right)$ provides the concept of reduced word (S-reduced in this text ( $\mathrm{S}$ from Serre), to avoid confusion with free group reduced), which makes the cycle construction the preferred option, most of the time.

A word of the form (5.2) is said to be $S$-reduced if the following two conditions hold:

- If $n=0$, then $g_{0} \neq 1$.

- If $1 \leq i<n$ and $e_{i+1}=\bar{e}_{i}$, then $g_{i} \notin G_{e_{i}} \tau_{e_{i}}$.

Note that S-reduced words play a major role in the theory of graphs of groups due to the following two well-known properties:

- every element of $\pi_{1}\left(\mathscr{E}, v_{0}\right) \backslash\{1\}$ can be represented by some S-reduced word,

- no S-reduced word represents the identity in $P(\mathscr{E})\left(\right.$ nor $\left.\pi_{1}\left(\mathscr{E}, v_{0}\right)\right)$.

It follows that the group $G_{v_{0}}$ (and therefore every vertex group) is naturally embedded into $\pi_{1}\left(\mathscr{H}, v_{0}\right)$.

HNN extensions and amalgamated free products arise as important particular cases of graphs of groups, by taking graphs with two edges, respectively of the form
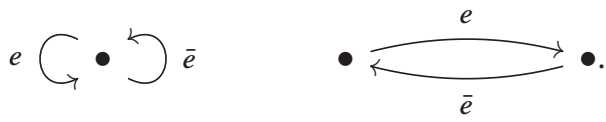

Moreover, whenever $\Gamma$ is finite, the fundamental group $\pi_{1}\left(\mathcal{E}, v_{0}\right)$ can be built from the vertex groups using a finite number of HNN extensions and amalgamated free products, where the associated/amalgamated subgroups are of the form $G_{e} \tau_{e}$. 
The nature of $\pi_{1}\left(\mathscr{G}, v_{0}\right)$ is conditioned by the nature of the vertex and edge groups. By a well-known theorem of Karrass, Pietrowski and Solitar [8] (see also [16, Theorem 7.3]), a finitely generated group is virtually free if and only if it is the fundamental group of a finite graph of finite groups. This important result provides the key to our main theorem:

Theorem 5.1. A finitely generated group admits a Stallings section if and only if it is virtually free.

Proof. Let $G$ be a finitely generated group. Assume that $S$ is a Stallings section for the m-epi $\pi: \widetilde{A}^{*} \rightarrow G$. We will show that the word problem submonoid $1 \pi^{-1}$ is context-free. By Muller-Schupp's Theorem [14], this is equivalent to $G$ being virtually free.

By the remark following the definition of Stallings section in Section 3, we can assume that $1 \in S_{1}$. Let $\theta: \widetilde{A}^{*} \rightarrow F_{A}$ denote the canonical morphism, as usual. We show that

$$
a_{1} \cdots a_{n} \in 1 \pi^{-1} \Longleftrightarrow S_{a_{1}} \cdots S_{a_{n}} \cap\left(1 \theta^{-1}\right) \neq \varnothing
$$

holds for all $a_{1}, \ldots, a_{n} \in \widetilde{A}$. Indeed, since $1 \in S_{g}$ if and only if $g=1$, we have $a_{1} \cdots a_{n} \in 1 \pi^{-1}$ if and only if $1 \in S_{\left(a_{1} \cdots a_{n}\right) \pi}$. By equation (3.3), this is equivalent to $1 \in \overline{S_{a_{1}} \cdots S_{a_{n}}}$, i.e.

$$
S_{a_{1}} \cdots S_{a_{n}} \cap\left(1 \theta^{-1}\right) \neq \varnothing .
$$

Therefore (5.3) holds.

We define now a transduction $\tau: \widetilde{A}^{*} \rightarrow 2^{\widetilde{A}^{*}}$ by

$$
\left(a_{1} \cdots a_{n}\right) \tau=S_{a_{1}} \cdots S_{a_{n}}
$$

for $a_{1}, \ldots, a_{n} \in \widetilde{A}$ and $1 \tau=1$. Since

$$
\Delta_{\tau}=\left\{\{a\} \times S_{a} \mid a \in \widetilde{A}\right\}^{*}
$$

is clearly a rational subset of $A^{*} \times A^{*}$, then $\tau$ is a rational transduction, and so must be $\tau^{-1}$.

Let $L=1 \theta^{-1}$. By Muller-Schupp's Theorem [14], $L$ is context-free and so $L \tau^{-1}$ is also context-free by Proposition 2.2.

By (5.3), we have

$$
a_{1} \cdots a_{n} \in 1 \pi^{-1} \Longleftrightarrow\left(a_{1} \cdots a_{n}\right) \tau \cap L \neq \emptyset \Longleftrightarrow a_{1} \cdots a_{n} \in L \tau^{-1}
$$

for all $a_{1}, \ldots, a_{n} \in \widetilde{A}$. Since $1 \in 1 \pi^{-1} \cap L \tau^{-1}$, it follows that $1 \pi^{-1}=L \tau^{-1}$ and is therefore context-free. By Muller-Schupp's Theorem, $G$ is virtually free. 
Conversely, assume that $G$ is virtually free. By the theorem ([8]) of Karrass, Pietrowski and Solitar, we may assume that $G=\pi_{1}\left(\mathscr{G}, v_{0}\right)$, where

$$
\mathcal{E}=\left(\left(G_{v}\right)_{v \in V},\left(G_{e}\right)_{e \in E},\left(\tau_{e}\right)_{e \in E}\right)
$$

is a graph of groups over a finite connected graph $\Gamma=\left(V, E, \tau,{ }^{-}\right)$, with finite vertex and edge groups.

For every $v \in V$, consider an alphabet $A_{v}=G_{v} \backslash\{1\}$ and take $A$ to be the disjoint union

$$
A=E \cup\left(\bigcup_{v \in V} A_{v}\right) .
$$

We shall consider the involutive alphabet $\widetilde{A}$, hence it is convenient to set

$$
e^{-1} \tau=\bar{e} \tau
$$

for every $e \in E$. For every $v \in V$, let $\varphi_{v}: \widetilde{A_{v}}{ }^{*} \rightarrow G_{v}$ be the canonical m-epi. Fix a spanning tree $T$ of $\Gamma$ and $v_{0} \in V$. We have a canonical m-epi $\varphi: \widetilde{A}^{*} \rightarrow \pi_{1}(\mathscr{E}, T)$. Composing with the canonical isomorphism $\pi_{1}(\mathscr{E}, T) \rightarrow \pi_{1}\left(\mathscr{E}, v_{0}\right)$, we obtain an m-epi $\widetilde{A}^{*} \rightarrow \pi_{1}\left(\mathcal{E}, v_{0}\right)$ which, by abuse of notation, shall also be denoted by $\varphi$.

We define $S \subseteq R_{A}$ to be the union of $R_{A_{v_{0}}}$ with the languages of the form

$$
\left(g_{0} \varphi_{v_{0}}^{-1}\right) e_{1}^{\varepsilon_{1}}\left(g_{1} \varphi_{v_{1}}^{-1}\right) \cdots e_{n}^{\varepsilon_{n}}\left(g_{n} \varphi_{v_{n}}^{-1}\right) \cap R_{A},
$$

where $\varepsilon_{i}= \pm 1, e_{i}^{\varepsilon_{i}} \tau=v_{i}, i=1, \ldots, n$, and $g_{0} e_{1}^{\varepsilon_{1}} g_{1} \cdots e_{n}^{\varepsilon_{n}} g_{n}$ is an S-reduced word of the form (5.2). We shall prove that $S$ is a Stallings section for the m-epi $\varphi: \widetilde{A}^{*} \rightarrow \pi_{1}\left(\mathscr{E}, v_{0}\right)$.

Since every element of $\pi_{1}\left(\mathscr{G}, v_{0}\right)$ can be represented by an S-reduced word, it follows easily that $S \varphi=\pi_{1}\left(\mathscr{E}, v_{0}\right)$. Since S-reduced words are well known to be closed under inversion, we have $S^{-1}=S$. Thus $S$ is a section for $\varphi$.

We may view $\Gamma$ as an $E$-automaton $\left(V, v_{0}, v_{0}, E^{\prime}\right)$ by taking

$$
E^{\prime}=\{(\bar{e} \tau, e, e \tau) \mid e \in E\} .
$$

The language of this automaton is precisely the set of closed paths with basepoint $v_{0}$, i.e. $C\left(\mathcal{G}, v_{0}\right)$. We define now a rational transducer $\left(V, v_{0}, v_{0}, E^{\prime \prime}\right)$ by replacing each label $e$ in the edges of $E^{\prime}$ by $\{e\} \times\left\{e, \bar{e}^{-1}\right\} A_{e \tau}^{*}$ (note that we must admit the double representation of edges when we go from $E$ to $\widetilde{E}$ ). This defines a rational transduction $\eta: E^{*} \rightarrow 2^{A^{*}}$. By Proposition 2.1 (ii), $\left(C\left(\mathcal{E}, v_{0}\right)\right) \eta$ is a rational language. Now it is easy to check that

$$
S=\left(A_{v_{0}}^{*}\left(C\left(\mathscr{G}, v_{0}\right)\right) \eta \backslash L\right) \cap R_{A},
$$

where $L$ denotes the language of all words in $A^{*}$ having some factor of the form

$$
\text { eue } e^{-1} \text { or } e u \bar{e}, \quad \text { with } u \in\left(G_{e} \tau_{e}\right) \varphi_{e \tau}^{-1} \text {, }
$$


for some $e \in E$. Note that the languages $\left(G_{e} \tau_{e}\right) \varphi_{e \tau}^{-1}$ are rational by Proposition 2.1 (iii). Since $R_{A}$ is also rational, it follows easily from Proposition 2.1 (i) that $S$ is a rational section for $\varphi$. Moreover, the construction of $S$ is effective.

Let $g \in \pi_{1}\left(\mathscr{E}, v_{0}\right)$. We must show that $S_{g}$ is an effectively constructible rational language. If $g \neq 1$, then it is well known that all the S-reduced words representing $g$ arise from the same closed path

$$
v_{0} \stackrel{e_{1}}{\longrightarrow} v_{1} \stackrel{e_{2}}{\longrightarrow} \cdots \stackrel{e_{n}}{\longrightarrow} v_{n}=v_{0} .
$$

As vertex groups are finite, it follows that there exist only finitely many S-reduced words representing $g$. Using the transduction built to prove the rationality of $S$, adapted in the obvious way, we deduce that $S_{g}$ is a finite union of rational languages, hence rational. If $g=1$, we get $S_{1}=1 \varphi_{v_{0}}^{-1} \cap R_{A_{v_{0}}}$, also rational in view of Proposition 2.1. All the constructions are effective, so $S_{g}$ is an effectively constructible rational language for every $g$.

Finally, let $g, h \in \pi_{1}\left(\mathscr{g}, v_{0}\right)$. We must show that $S_{g h} \subseteq \overline{S_{g} S_{h}}$. Note that the mapping $\xi$ defined above for S-reduced words extends naturally to $S$, taking values on the free monoid on $E$ (identifying $e^{-1}$ with $\bar{e}$ ).

Fix $u \in S_{g}$ and $w \in S_{h}$. We can compute some word ( $\left.u w\right) \psi \in S_{g h}$ by successively lifting the substitutions

$$
e\left(g \tau_{e}\right) \bar{e} \rightarrow g \tau_{\bar{e}} \quad\left(e \in E, g \in G_{e}=G_{\bar{e}}\right) .
$$

Note that $(u w) \psi$ is not unique, so we fix one of the possible choices. We may write $u \xi=u^{\prime} p, w \xi=p^{-1} w^{\prime}$ and $(u w) \psi \xi=u^{\prime} w^{\prime}$ for some $u^{\prime}, w^{\prime}, p$. Indeed, we may assume that $\psi: S \times S \rightarrow S$ is a mapping defined in the above terms.

Without loss of generality, we shall assume that $u^{\prime}, w^{\prime} \neq 1$. The remaining cases constitute mere simplifications of this general case.

Let $z \in S_{g h}$. Then $z \xi=u^{\prime} w^{\prime}$. If we compute $\left(z w^{-1}\right) \psi$, this implies that we must remove precisely $\left|w^{\prime} \xi\right|$ edges from each of the words $z$ and $w^{-1}$, hence the prefixes of $z$ and $\left(z w^{-1}\right) \psi$ ending at edge number $\left|u^{\prime}\right|$ must coincide. Denote this prefix by $z_{1}$ and write $\left(z w^{-1}\right) \psi=z_{1} s_{1}$. Similarly, the suffixes of $z$ and $\left(u^{-1} z\right) \psi$ starting at edge number $\left|w^{\prime} \xi\right|$ (counting in reverse order) must also coincide. Denote this by $z_{2}$ and write $\left(u^{-1} z\right) \psi=s_{2} z_{2}$. Now we have $\left(z w^{-1}\right) \psi \xi=u^{\prime} p$ and $\left(u^{-1} z\right) \psi \xi=p^{-1} w^{\prime}$, hence on computing $x=\left(\left(z w^{-1}\right) \psi\left(u^{-1} z\right) \psi\right) \psi \in S_{g h}$ we must cancel $p$ edges from each word. It follows that $s_{2} \xi=\left(s_{1} \xi\right)^{-1}$ and the words $x$ and $z$ differ at most in the factor between the occurrence of edge number $\left|u^{\prime}\right|$ and the next edge.

Write $z=z_{1} q z_{2}$. Note that, since every element of a vertex group has finite order and we have at our disposal an involutive alphabet, we can always, if necessary, replace the last vertex component of $s_{1}$ by an equivalent word so that $s_{1}^{-1} q$ is reduced. 
Let $y=s_{1}^{-1} q z_{2}$. We have

$$
y \varphi=\left(s_{1}^{-1} q z_{2}\right) \varphi=\left(s_{1}^{-1} z_{1}^{-1} z\right) \varphi=\left(w z^{-1} z\right) \varphi=w \varphi=h .
$$

Since $w \in S_{h}$ arises from an S-reduced word, it follows that $|w \xi|$ is minimum possible among $\left\{|t \xi| \mid t \in h \varphi^{-1}\right\}$ and so

$$
|y \xi| \geq|w \xi|=\left|\left(u^{-1} z\right) \psi \xi\right|=\left|s_{2} \xi\right|+\left|z_{2} \xi\right|=\left|s_{1}^{-1} \xi\right|+\left|z_{2} \xi\right|=|y \xi|,
$$

whence $|y \xi|=|w \xi|$. It follows from minimality that the word $y$ must arise from an S-reduced word. Since $y \in R_{A}$ by our preprocessing of $s_{1}$ (recall also that the first letter of $z_{2}$ is in $\widetilde{E}$ ), it follows that $y \in S$ and so $y \in S_{h}$. Therefore

$$
z=z_{1} q z_{2}=\overline{z_{1} s_{1} s_{1}^{-1} q z_{2}}=\overline{\left(\left(z w^{-1}\right) \psi\right) y} \in \overline{S_{g} S_{h}}
$$

and so we have $S_{g h} \subseteq \overline{S_{g} S_{h}}$. This completes the proof that $S$ is a Stallings section for $\varphi$.

\section{Sections with good properties}

Having established that finitely generated virtually free groups are precisely the groups with a Stallings section, we shall now discuss the possibility of imposing stronger conditions on their Stallings sections, with the purpose of allowing further applications of the Stallings automata $\Gamma(G, H, \pi) \sqcap S$.

We start with the concept of extendable Stallings section, which will turn out to be useful to characterize finite index subgroups.

Let $S$ be a Stallings section for the m-epi $\pi: \widetilde{A}^{*} \rightarrow G$. We say that $S$ is extendable if, for every $u \in S$, there exists some $w \in R_{A}$ such that $u w^{*} \subseteq S$ and

$$
u \in \operatorname{Pref}\left(S_{\left(u w^{n} u^{-1}\right) \pi}\right)
$$

for almost all $n \in \mathbb{N}$.

Proposition 6.1. Every finitely generated virtually free group has an extendable Stallings section.

Proof. Let $G$ be a finitely generated virtually free group. Assume first that $G$ is finite. Let $\pi: \widetilde{A}^{*} \rightarrow G$ be an m-epi. By the proof of Proposition 3.2, we may take $S=R_{A}$ and $w=1$ for every $u \in S$. Hence $u w^{*} \subseteq S$. We have $S_{g}=\overline{g \pi^{-1}}$ for every $g \in G$. Next we show that $\operatorname{Pref}\left(S_{g}\right)=R_{A}$.

Let $z \in R_{A}$ and take $a \in \widetilde{A}$ such that $z a \in R_{A}$. Since $G$ is finite, there exists some $m \in \mathbb{N}$ such that every element of $G$ can be represented by some word of length $<m$. In particular, there exists some $x \in R_{A}$ such that $\left(\left(a^{-m} z^{-1}\right) \pi\right) g=x \pi$ and $|x|<m$. Hence $\left(z a^{m} x\right) \pi=g$ and so $\overline{z a^{m} x} \in \overline{g \pi^{-1}}=S_{g}$. Since $z a^{m} \in R_{A}$ and $|x|<m$, we get $z \in \operatorname{Pref}\left(S_{g}\right)$ and $\operatorname{so} \operatorname{Pref}\left(S_{g}\right)=R_{A}$. 
Therefore condition (6.1) holds and so $R_{A}$ is an extendable Stallings section for $\pi: \widetilde{A}^{*} \rightarrow G$ when $G$ is finite. Let us assume from now on that $G$ is infinite.

By the theorem of Karrass, Pietrowski and Solitar, we have that $G=\pi_{1}\left(\mathscr{E}, v_{0}\right)$, where

$$
\mathcal{E}=\left(\left(G_{v}\right)_{v \in V},\left(G_{e}\right)_{e \in E},\left(\tau_{e}\right)_{e \in E}\right)
$$

is a graph of groups over a finite connected graph $\Gamma=\left(V, E, \tau,{ }^{-}\right)$, with finite vertex and edge groups. Moreover, we may assume that none of the boundary monomorphisms $\tau_{e}$ is surjective. Indeed, if $\tau_{e}$ were surjective, then $G_{e \tau} \cong G_{e}$ would embed in $G_{\bar{e} \tau}$ and we could replace our graph of groups by a graph of groups with isomorphic fundamental group and one less vertex.

We consider the same m-epi $\varphi: \widetilde{A}^{*} \rightarrow G$ of the proof of the opposite implication in Theorem 5.1 and the same Stallings section $S$.

Let $u \in S$. If $u \notin R_{A_{v_{0}}}$, write $u=u^{\prime} e^{\varepsilon} u^{\prime \prime}$ with $u^{\prime \prime} \in R_{A_{v_{0}}}$. Then there exists some $x \in R_{A_{v_{0}}}$ such that $u^{\prime \prime} x \in R_{A_{v_{0}}} \backslash G_{e} \tau_{e^{\varepsilon}} \varphi_{v_{0}}^{-1}$. This follows from our assumption on the nonsurjectivity of the boundary maps, and also from the fact (remarked in the proof of Theorem 5.1 above) that we can always change the first/last letter of a representative of an element of a vertex group. On the other hand, let $y$ be an element of $R_{A_{e}-\varepsilon_{\tau}} \backslash G_{e} \tau_{e^{-\varepsilon}} \varphi_{e^{-\varepsilon_{\tau}}}^{-1}$ and write $w=x e^{-\varepsilon} y e^{\varepsilon} u^{\prime \prime}$. It is routine to check that $u w^{*} \subseteq S$ and (6.1) holds (only finitely many of the edge letters can be affected by multiplying by $u^{-1}$ on the right).

If $u \in R_{A_{v_{0}}}$, we proceed with a straightforward adaptation of the preceding case, using any edge of the form $v_{0} \stackrel{e}{\rightarrow} v_{1}$ ( $\Gamma$ must have edges since we are assuming $G$ to be infinite).

Therefore $S$ is extendable as claimed.

We can now derive the following application of the concept of extendable Stallings section:

Theorem 6.2. Let $S$ be an extendable Stallings section for the m-epi $\pi: \widetilde{A}^{*} \rightarrow G$ and let $H$ be a finitely generated subgroup of $G$. Then the following conditions are equivalent:

(a) $H$ has finite index in $G$,

(b) $S \subseteq \operatorname{Pref}\left(S_{H}\right)$,

(c) every word of $S$ labels a path out of the basepoint of $\Gamma(G, H, \pi) \sqcap S$.

Proof. (a) $\Rightarrow$ (b) Suppose that $u \in S \backslash \operatorname{Pref}\left(S_{H}\right)$. Since $S$ is extendable, there exist some $v \in R_{A}$ and $m \in \mathbb{N}$ such that $u v^{*} \subseteq S$ and $u \in \operatorname{Pref}\left(S_{\left(u v^{n} u^{-1}\right) \pi}\right)$ for $n \geq m$. We claim that

$$
H\left(u v^{j}\right) \pi \neq H\left(u v^{i}\right) \pi \quad \text { if } j \geq i+m .
$$


Indeed, assume that $j \geq i+m$. If $H\left(u v^{j}\right) \pi=H\left(u v^{i}\right) \pi$, then $\left(u v^{j-i} u^{-1}\right) \pi \in H$ and so

$$
u \in \operatorname{Pref}\left(S_{\left(u v^{j-i} u^{-1}\right) \pi}\right) \subseteq \operatorname{Pref}\left(S_{H}\right),
$$

a contradiction. Therefore (6.2) holds and so $H$ has infinite index in $G$.

(b) $\Rightarrow$ (c) This implications is clear since $S_{H} \subseteq L(\Gamma(G, H, \pi) \sqcap S)$ by Theorem 3.9.

(c) $\Rightarrow$ (a) Assume now that every word of $S$ labels a path out of the basepoint $q_{0}$ of $\mathcal{A}=\Gamma(G, H, \pi) \sqcap S$. Let $Q$ denote the (finite) vertex set of $\mathcal{A}$. For every $q \in Q$, fix a path

$$
q_{0} \stackrel{w_{q}}{\longrightarrow} q
$$

We claim that

$$
G=\bigcup_{q \in Q} H\left(w_{q} \pi\right)
$$

Indeed, let $g \in G$, and take $u \in S_{g}$. Then there is a path in $\mathcal{A}$ of the form $q_{0} \stackrel{u}{\rightarrow} q$ for some $q \in Q$. Hence $u w_{q}^{-1} \in L(\mathcal{A}) \subseteq H \pi^{-1}$ by Theorem 3.9 and so we have $g=u \pi \in H\left(w_{q} \pi\right)$. Thus (6.3) holds and so $H$ has finite index in $G$.

A natural question to ask is whether or not one could replace condition (S2) in the definition of Stallings section by the stronger condition

(S2') $S_{g h}=\overline{S_{g} S_{h}}$ for all $g, h \in G$.

However, we can prove that this condition can only be assumed in the simplest cases:

Proposition 6.3. The following conditions are equivalent for a group $G$ :

(a) there exist an m-epi $\pi: \widetilde{A}^{*} \rightarrow G$ and a Stallings section $S$ for $\pi$ satisfying condition (S2'),

(b) $G$ is either finite or free of finite rank,

(c) $R_{A}$ is a Stallings section for some m-epi $\pi: \widetilde{A}^{*} \rightarrow G$.

Proof. (a) $\Rightarrow$ (b) Let $S$ be a Stallings section $S$ for $\pi: \widetilde{A}^{*} \rightarrow G$ satisfying (S2'). Then $S_{1}^{-1}=S_{1}=\overline{S_{1}^{2}}$ and so we can view $\left(S_{1}, \circ\right)$ as a subgroup of $\left(R_{A}, \circ\right) \cong F_{A}$, where $u \circ v=\overline{u v}$. The same holds for $(S, \circ)$ since $S^{-1}=S=\overline{S^{2}}$, and $\left(S_{1}, \circ\right)$ is then a subgroup of $(S, \circ)$. Now $(S, \circ)$ must be free by Nielsen's Theorem. Since $S$, being a Stallings section, is rational, so is $(S, \circ)$ (a rational expression for $S$ as a subset of $\widetilde{A}^{*}$ translates through reduction to a rational expression for $S$ as a subset of $\left.\left(R_{A}, \circ\right)\right)$. The same happens with $S_{1}$, so it follows from Anisimov and Seifert's Theorem [1, Theorem 3.1] that both $(S, \circ)$ and $\left(S_{1}, \circ\right)$ are finitely generated groups. Hence $(S, \circ)$ is a free group of finite rank. 
For every $u \in S$, we have

$$
\overline{u S_{1} u^{-1}} \subseteq \overline{S_{u \pi} S_{1} S_{u^{-1} \pi}}=S_{1},
$$

hence $\left(S_{1}, \circ\right)$ is a finitely generated normal subgroup of the free group $(S, \circ)$. By [11, Proposition 3.12], $\left(S_{1}, \circ\right)$ is either trivial or has finite index in $(S, \circ)$. On the other hand, we claim that

$$
\overline{u S_{1}}=\overline{v S_{1}} \Longleftrightarrow u \pi=v \pi
$$

holds for all $u, v \in S$. The direct implication follows from $S_{1} \pi=1$. Conversely, assume that $u \pi=v \pi$. Then

$$
\overline{v^{-1} u} \in \overline{S_{v^{-1} \pi} S_{u \pi}}=S_{1}
$$

and so $u \in \overline{v S_{1}}$ and $\overline{u S_{1}} \subseteq \overline{v S_{1}}$. By symmetry, we get $\overline{u S_{1}}=\overline{v S_{1}}$ and so (6.4) holds.

It is now straightforward to check that

$$
(S, \circ) /\left(S_{1}, \circ\right) \rightarrow G, \quad \overline{u S_{1}} \mapsto u \pi,
$$

is a group isomorphism. Hence either $G \cong(S, \circ)$ is a free group of finite rank, or $G \cong(S, \circ) /\left(S_{1}, \circ\right)$ is a finite group.

(b) $\Rightarrow$ (c) Immediate from the proof of Proposition 3.2.

(c) $\Rightarrow$ (a) Assume that $S=R_{A}$ is a Stallings section for the m-epi $\pi: \widetilde{A}^{*} \rightarrow G$. Let $u \in S_{g}$ and $v \in S_{h}$ for some $g, h \in G$. Since $\overline{u v} \pi=(u v) \pi=g h$, we get $\overline{u v} \in S_{g h}$ and so $\overline{S_{g} S_{h}} \subseteq S_{g h}$. Therefore $S_{g h}=\overline{S_{g} S_{h}}$ and so $R_{A}$ satisfies condition (S2').

Acknowledgments. We are grateful to the anonymous referee for suggesting substantial simplifications of the proof of Theorem 5.1 with respect to the original version.

\section{Bibliography}

[1] L. Bartholdi and P. V. Silva, Rational subsets of groups, preprint (2010), http: // arxiv. org/abs/1012.1532; Chapter 23 of the handbook AutoMathA.

[2] M. Benois, Parties rationnelles du groupe libre, C. R. Acad. Sci. Paris 269 (1969), 1188-1190.

[3] J. Berstel, Transductions and Context-Free Languages, Teubner, Stuttgart, 1979.

[4] T. H. Cormen, C.E. Leierson, R. L. Rivest and C. Stein, Introduction to Algorithms, 2nd ed., The MIT Press, Cambridge, 2001. 
[5] R. H. Gilman, S. Hermiller, D. F. Holt and S. Rees, A characterisation of virtually free groups, Arch. Math. 89 (2007), no. 4, 289-295.

[6] I. Kapovich and A. Miasnikov, Stallings foldings and subgroups of free groups, J. Algebra 248 (2002), 608-668.

[7] I. Kapovich, R. Weidmann and A. Miasnikov, Foldings, graphs of groups and the membership problem, Internat. J. Algebra Comput. 15 (2005), no. 1, 95-128.

[8] A. Karrass, A. Pietrowski and D. Solitar, Finitely generated groups with a free subgroup of finite index, J. Aust. Math. Soc. 16 (1973), 458-466.

[9] M. Ladra and P. V. Silva, The generalized conjugacy problem for virtually free groups, Forum Math. 23 (2011), 447-482.

[10] M. Lohrey and G. Sénizergues, When is a graph product of groups virtually free?, Comm. Algebra 35 (2007), no. 2, 617-621.

[11] R. C. Lyndon and P. E. Schupp, Combinatorial Group Theory, Springer, Berlin, 1977.

[12] L. Markus-Epstein, Stallings foldings and subgroups of amalgams of finite groups, Internat. J. Algebra Comput. 17 (2007), no. 8, 1493-1535.

[13] A. Miasnikov, E. Ventura and P. Weil, Algebraic extensions in free groups, in: Geometric Group Theory, Trends Math., Birkhäuser, Basel (2007), 225-253.

[14] D. E. Muller and P.E. Schupp, Groups, the theory of ends, and context-free languages, J. Comput. System Sci. 26 (1983), no. 3, 295-310.

[15] G. Nivasch, Improved bounds and new techniques for Davenport-Schinzel sequences and their generalizations, J. ACM 57 (2010), Article No. 17.

[16] P. Scott and T. Wall, Topological methods in group theory, in: Homological Group Theory (Durham 1977), London Math. Soc. Lecture Note Ser. 36, Cambridge University Press, Cambridge (1979), 137-203.

[17] J.P. Serre, Arbres, Amalgames, $\mathrm{SL}_{2}$, Astérisque 46, Société Mathématique de France, Paris, 1977; translation: Trees, Springer Monogr. Math., Springer, Berlin, 2003.

[18] P. V. Silva, Fixed points of endomorphisms of virtually free groups, Pacific J. Math. 263 (2013), no. 1, 207-240.

[19] J. Stallings, Topology of finite graphs, Invent. Math. 71 (1983), 551-565.

[20] R. E. Tarjan, Efficiency of a good but not linear set merging algorithm, J. ACM 22 (1975), no. 2, 215-225.

[21] W. M. Touikan, A fast algorithm for Stallings' folding process, Internat. J. Algebra Comput. 16 (2006), no. 6, 1031-1046. 
Received November 27, 2014; revised May 22, 2015.

\section{Author information}

Pedro V. Silva, Centro de Matemática, Faculdade de Ciências, Universidade do Porto, R. Campo Alegre 687, 4169-007 Porto, Portugal.

E-mail: pvsilva@fc.up.pt

Xaro Soler-Escrivà, Dpt. de Matemàtiques, Universitat d'Alacant, Apartat de correus 99, 03080-Alacant, Spain.

E-mail: xaro.soler@ua.es

Enric Ventura, Dept. Mat. Apl. III, Universitat Politècnica de Catalunya, Manresa, Barcelona, Catalunya, Spain.

E-mail: enric.ventura@upc.edu 